\title{
Using X-ray catalogues to find counterparts to unassociated high-energy Fermi/LAT sources
}

\section{R. Landi*}

INAF - IASF Bologna

E-mail: landidiasfbo.inaf.it

\section{Bassani}

INAF - IASF Bologna

E-mail: bassani@iasfbo.inaf.it

\section{J. B. Stephen}

INAF - IASF Bologna

E-mail: stephen@iasfbo.inaf.it

\section{N. Masetti}

INAF - IASF Bologna

E-mail: masetti@iasfbo.inaf.it

\section{A. Malizia}

INAF - IASF Bologna

E-mail: malizia@iasfbo.inaf.it

\section{P. Ubertini}

INAF - IAPS Rome

E-mail: pietro.ubertini@iaps.inaf.it

The first Fermi Large Area Telescope (LAT) catalogue of sources (1FHL) emitting at high energies (above $10 \mathrm{GeV}$ ) reports the details of 514 objects detected in the first three years of the Fermi mission. Of these, 71 were reported as unidentified in the 1FHL catalogue, although six are likely to be associated with a supernova remnant (SNR), a Pulsar Wind Nebula (PWN) or a combination of both, thereby leaving a list of 65 still unassociated objects. Herein, we report a preliminary analysis on this sample of objects concentrating on nine 1FHL sources, which were found to have a clear optical extragalactic classification. They are all blazar, eight BL Lac and one flat spectrum radio quasar, typically at redshift greater than 0.1 .

Swift: 10 Years of Discovery,

2-5 December 2014

La Sapienza University, Rome, Italy

\footnotetext{
* Speaker.
} 


\section{Introduction}

We report on a preliminary work that is part of a larger programme aimed at id entifying unassociated sources in the first Fermi/LAT high-energy catalogue [1FHL, 1], which contains 514 objects detected above $10 \mathrm{GeV}$. The majority of these sources are identified with known objects (449 or $87 \%$ of the sample): approximately $75 \%$ with AGNs (mostly blazars), while Galactic sources (pulsars, PWNs, SNRs, high-mass binaries, and star-forming regions) collectively represent $10 \%$ of the sample. The fraction of unassociated sources is less than $14 \%$ corresponding to 71 objects, of which six are likely to be associated with a SNR, a PWN or a combination of both, thereby leaving a list of 65 still unidentified objects. The third Fermi/LAT catalogue [3FGL, 2], which has recently been published, contains most of these unassociated 1FHL sources except for 13 objects that are missing. The main motivation behind the 1FHL catalogue was to find the hardest gammaray sources in the sky and to get a sample of objects which are good candidate for detection at $\mathrm{TeV}$ energies.

As a first step, we have cross-correlated the sample of 65 objects with both the ROSAT Bright (RASSBSC, [3]) and the XMM-Newton Slew Survey [4] catalogues, following the prescription of [5] and finding the likely counterpart to 19 1FHL sources. Secondly, we have extended our analysis using data collected with the X-ray telescope (XRT) on-board Swift [6]; this was done by cross-correlating the list of unassociated 1FHL sources with all the XRT archival data up to the end of 2014 and found to be within around 10 arcmin from the Fermi best-fit position. This analysis has led us to investigate a further set of sources, increasing the sample for which a likely association is found to around 30, i.e. half of the original set of objects. The remaining 1FHL sources have also been investigated on an individual basis. The nature of each likely counterpart has been studied by means of a multi-waveband approach using information in the radio, infrared, and optical wavebands. In particular, we use the WISE colours as discussed by [7] to test the possible blazar nature of each source: these authors found that in the $W 2-W 3$ versus $W 1-W 2$ colour-colour plot, the positions of gamma-ray emitting blazars are all within a well-defined region known as the "Blazar strip".

Herein, we report some results from this on going programme concentrating on nine 1FHL objects, which were found to have an optical classification. All sources have a counterpart in the third Fermi/LAT catalogue and the same association we found in this work, although two display multiple X-ray counterparts and other two an X-ray detection outside the 1FHL 95\% positional uncertainty.

\subsection{Objects classified optically}

Nine of the various sources analysed in the project were found to have a likely association with known blazars: they are listed in Table 1, where we report the Fermi name, the coordinates of the associated soft X-ray counterpart we found (from either ROSAT Bright, XMM-Newton Slew or Swift/XRT observations), the X-ray error radius, the source optical class, and the redshift when available. In the following, we describe briefly each individual source.

The soft X-ray counterpart to 1FHL J0110.0-4023 is associated with RBS0158 (also ATESP J010956-402051) a radio source that shows 20 and $36 \mathrm{~cm}$ flux densities of 57 and $36 \mathrm{mJy}$, respectively [8]. The source, which was optically classified as a BL Lac object by [9], has a red- 
Table 1: Unidentified Fermi 1FHL sources with an RASSBSC/XMMSlew/XRT counterpart.

\begin{tabular}{|c|c|c|c|c|c|}
\hline \multirow[t]{2}{*}{ Fermi Name } & \multicolumn{2}{|c|}{$\mathrm{X}$-ray counterpart } & \multirow{2}{*}{$\begin{array}{c}\text { X-ray error }{ }^{\dagger} \\
(\operatorname{arcsec})\end{array}$} & \multirow[t]{2}{*}{ Catalogue } & \multirow[t]{2}{*}{ Optical class $(z)$} \\
\hline & R.A.(J2000) & Dec.(J2000) & & & \\
\hline 1FHL J0110.0-4023 & 010956.5 & -402047.0 & 7.0 & RASSBSC & BL Lac (0.313) \\
\hline 1FHL J0118.5-1502 & 011905.4 & -145906.0 & 14.0 & RASSBSC & BL Lac (0.1147) \\
\hline 1FHL J0601.0+3838 & 060102.7 & +383827.2 & 5.2 & XRT & BL Lac \\
\hline 1FHL J0828.9+0902 & 082930.1 & +085820.5 & 4.2 & XRT & FSRQ (0.866) \\
\hline 1FHL J0841.2-3556 & 084121.6 & -355550.8 & 6.0 & XRT & BL Lac $(\geq 0.15)$ \\
\hline \multirow[t]{2}{*}{ 1FHL J1353.0-6642 } & 135341.1 & -664002.0 & 8.0 & RASSBSC & BL Lac $(\geq 0.15)$ \\
\hline & 135340.6 & -663958.0 & 3.0 & XMMSlew & - \\
\hline 1FHL J1406.4+1646 & 140659.2 & +164206.0 & 3.7 & XRT & BL Lac $(\geq 0.623)$ \\
\hline \multirow[t]{2}{*}{ 1FHL J1440.6-3847 } & 144037.4 & -384658.5 & 7.0 & RASSBSC & BL Lac \\
\hline & 144038.1 & -384653.8 & 3.0 & XMMSlew & - \\
\hline 1FHL J2004.7+7003 & 200504.5 & +7004 40.6 & 4.0 & XMMSlew & BL Lac \\
\hline
\end{tabular}

$\dagger$ ROSAT, XMM-Newton Slew errors are $1 \sigma$ radius, while Swift/XRT errors are $1.6 \sigma$ radius; ${ }^{\ddagger}$ Object at low Galactic latitude, i.e. within \pm 10 degrees of the Galactic plane.

shift of 0.313. It is also listed in the WISE catalogue [10] with colours $W 2-W 3=1.84$ and $W 1-W 2=0.59$, which are well inside the blazar strip.

The small XRT error circle of 1FHL J0601.0+3838 allows the identification of the X-ray source with the bright radio object B20557+38, which displays 20 and $92 \mathrm{~cm}$ flux densities of 704 and $1882 \mathrm{mJy}$, respectively. This object is reported in various radio archives and has a radio spectrum with index of $\sim 0.7$ (see NASA/IPAC Extragalactic Database, NED). The source has WISE colours $W 2-W 3=2.47$ and $W 1-W 2=0.97$, typical of gamma-ray emitting blazars. It was optically classified as a BL Lac by [11].

For 1FHL J1353.0-6642, the restricted X-ray position provides a secure identification with VASC J1353-66. This object, which is listed in the XMM-Newton Slew Survey, has an X-ray 0.2$12 \mathrm{keV}$ flux of $3.9 \times 10^{-12} \mathrm{erg} \mathrm{cm}^{-2} \mathrm{~s}^{-1}$. It is detected in radio at various frequencies, including the $36 \mathrm{~cm}$ one (flux density of $70.7 \mathrm{mJy}$, see [12]), and shows a flat radio spectrum (see [13]), while it is not detected by WISE. The source was optically classified as a BL Lac by [13], while [14] were able to put a lower limit of 0.15 to the source redshift.

The X-ray counterpart to 1FHL J1440.6-3847 is unambiguously identified with the galaxy

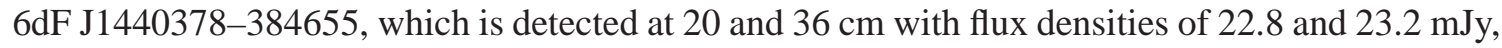
respectively; the $0.2-12 \mathrm{keV}$ flux is $7.9 \times 10^{-12} \mathrm{erg} \mathrm{cm}^{-2} \mathrm{~s}^{-1}$. The WISE colours $(W 2-W 3=1.38$ and $W 1-W 2=0.62$ ) locate the source outside the blazar strip. 6dF J1440378-384655 is classified as a BL Lac in NED (see also [15]), but on the basis of a poor quality optical spectrum.

The soft X-ray counterpart to 1 FHL J2004.7+7003 is radio detected at $20 \mathrm{~cm}$ with a flux density of $6.5 \mathrm{mJy}$ and listed in the WISE catalogue with colours $W 2-W 3=2.21$ and $W 1-$ $W 2=0.77$, i.e. fully compatible with the blazar strip. It is variable in both WISE ( $W 1$ and $W 2$ wavebands) and XMM-Newton Slew catalogues: the X-ray $0.2-12 \mathrm{keV}$ flux ranges from 2.8 to $8.2 \times 10^{-12} \mathrm{erg} \mathrm{cm}^{-2} \mathrm{~s}^{-1}$. This source was studied and discussed by various authors: all suggested that it is probably a BL Lac (see [16];[17];[18]), as confirmed in the 3FGL catalogue.

Four cases deserve a more in-depth analysis because they have multiple X-ray counterparts or have an association located outside the 1FHL positional uncertainty.

The only X-ray source we found in the case of 1FHL J0118.5-1502 is a bright ROSAT source, 


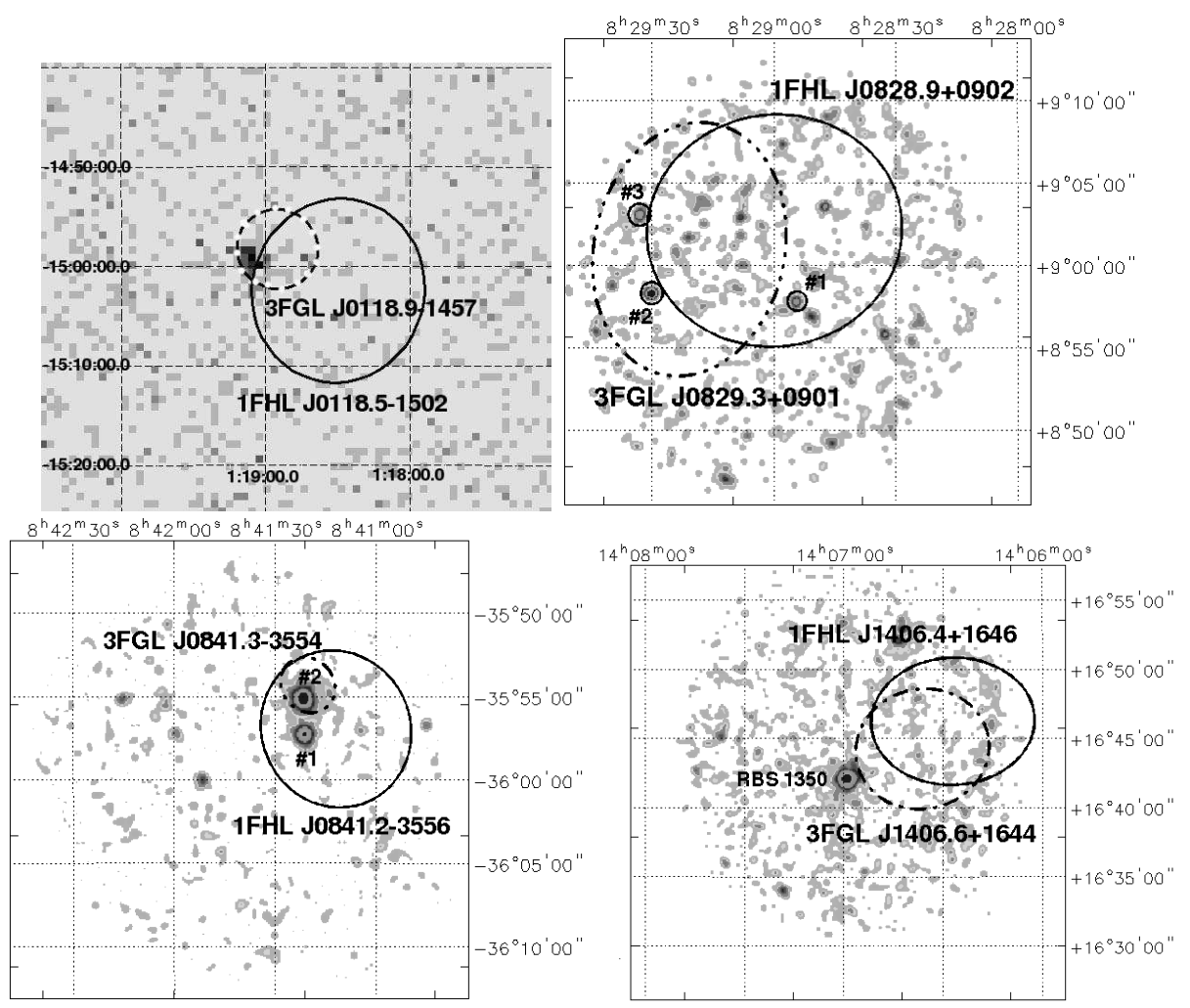

Figure 1: X-ray images of 1FHL J0118.5-1502 (ROSAT, upper left panel), 1FHL J0841.2-3556 (XRT, upper right panel), 1FHL J0828.9+0902 (XRT, bottom left panel), and 1FHL 1406.4+1646 (XRT, bottom right panel). The black ellipse and the black-dotted ellipse depict the positional uncertainty of the 1FHL and 3FGL sources, respectively. See details in the text.

which is located just outside the border of the Fermi error ellipse (left upper panel of Figure 1). Despite this, the source is within the positional uncertainty quoted for the 3FGL counterpart. The ROSAT source, which has the greatest error radius reported in Table 1, has a radio association in the NVSS (NVSS J011904-145858) with a $20 \mathrm{~cm}$ flux density of $5 \mathrm{mJy}$ and is WISE-detected with colours $W 1-W 2=0.521$ and $W 2-W 3=1.713$. The source was optically studied by [15] and found to display a spectrum with only absorption lines: it was therefore classified as a BL Lac at redshift 0.1147 .

In the case of 1FHL J0828.9+0902, various soft X-ray sources are found inside or at the border of the 1FHL error ellipse (right upper panel of Figure 1). Source \#1 is not detected in radio and has WISE colours that are not compatible with a blazar classification. Despite this, it is listed in NED as a QSO candidate (SDSS J082854.54+085751.2) at $z=0.855$ (see [19]). Similarly, source \#3 is not detected at radio frequencies, and it does not show WISE colours compatible with those typically displayed by blazars. The remaining object (source \#2) coincides with the radio source NVSS J082930+085821 (also TXS 0826+091), which displays a $20 \mathrm{~cm}$ flux density of $333.9 \mathrm{mJy}$. This X-ray detection is identified with a QSO at $z=0.866$ in NED. It was also classified as a flat spectrum radio object by [20], but its WISE colours $(W 2-W 3=3.08$ and $W 1-W 2=0.66)$ are outside the blazar strip. Source \#2 is also the association reported in the $3 F G L$ catalogue and it 


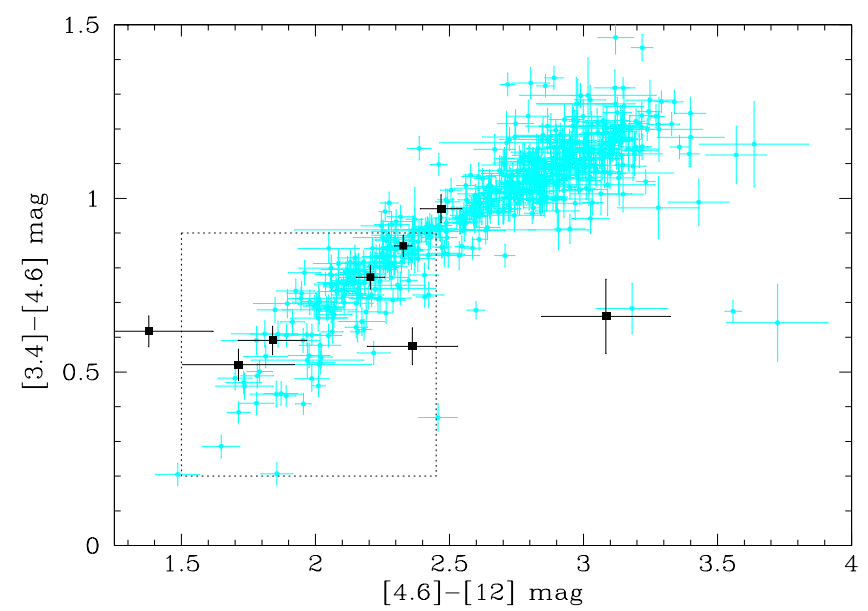

Figure 2: The [4.6]-[12]/[3.4]-[4.6] MIR colour-colour plot reporting the positions of gamma-ray emitting blazars (in cyan) associated with WISE sources forming the blazar strip (see [7] for more details), together with the BL Lac objects (filled squares) identified in this paper.

looks like the most promising one at the moment.

The error box of 1FHL J0841.4-3558 contains two X-ray sources, as evident in the left lower panel of Figure 1: one is a bright ROSAT/XRT object (R.A.(J2000) $=08^{h} 41^{m} 21^{\prime} .40$ and Dec.(J2000) $=-35^{\circ} 57^{\prime} 04^{\prime \prime} .50,9$ arcsec error radius) associated with the star 2MASS J084121323557154 (also HIP 42640) of spectral type F2V, which is unlikely to emit gamma-rays. The other, reported in Table 1, is located only 1.3 arcmin north of the star; it is listed as a radio source in various catalogues and has WISE colours $W 2-W 3=2.328$ and $W 1-W 2=0.863$, which locate the source in the locus of gamma-ray blazars. The optical spectrum obtained recently by [14] is featureless and the source was classified as a BL Lac at $z>0.15$. Note that this source is still listed as an unclassified blazar in the third Fermi/LAT catalogue.

In the case of 1 FHL J1406.4+164, the only X-ray detection is just 3 arcmin outside the Fermi 1FHL error ellipse, but it is located at the border of the positional uncertainty of 3FGL J1406.6+1644 (right lower panel of Figure 1). This X-ray object is associated with RBS 1350, which is classified as a BL Lac object and suggested to be an extreme high-energy peaked blazar or a TeV candidate (see [21]; [22]). The source redshift has a lower limit of 0.623 and a photometric value of 1.985 . In radio, the source has a $20 \mathrm{~cm}$ flux density around $78 \mathrm{mJy}$, while its WISE colours $(W 2-W 3=2.362$ and $W 1-W 2=0.574)$ are compatible with the blazar strip. Given the overall properties RBS 1350 and the overlap in positional uncertainties between the 1FHL and the 3FGL sources, we regard the association proposed here as likely, although not certain.

\section{Discussion and conclusions}

The main result of this work is that we have associated nine unidentified 1FHL sources with blazars, eight of the BL Lac type and one of the Flat Spectrum Radio Quasar type. Another interesting result is that all these sources are at redshift higher than 0.1 and hence allow probing the BL Lac population at a further distance than usual. The third evidence, coming from this work, is that all our BL Lacs are good candidates to be TeV emitting objects. As discussed by [14], and 
in the references therein, the $\mathrm{TeV}$ emitting BL Lacs populate a well-defined region of the WISE colour-colour diagram, i.e a square located in the lower part of the blazar strip. Therefore, objects with colours compatible with the $\mathrm{TeV}$ square are good candidates to emit at $\mathrm{TeV}$ energies. The WISE colour-colour diagram for the objects listed in Table 1, for which we have WISE colours, is plotted in Figure 2: as expected, all BL Lacs lie within or nearby the limits of the locus populated by $\mathrm{TeV}$-emitting BL Lacs and therefore they are good candidate for very high-energy observations; the only exception is the FSRQ (1FHL J0828.9+0902), which is even located outside the blazar strip. Further results stemming from the analysis described above are being prepared, and an optical follow-up program of the associations we found is well underway with time already assigned at various telescopes. Overall, the results obtained so far validate the goodness of our analysis method, which can be applied to the much larger set of still unidentified sources in the 3FGL catalogue or in further high-energy Fermi catalogues.

\section{References}

[1] M. Ackermann et al., The first Fermi-LAT catalog of sources above 10 GeV, ApJS 209 (2013) 34

[2] F. Acero et al., Fermi Large Area Telescope third source catalogue, ApJS 218 (2015) 23

[3] W. Voges et al., The ROSAT all-sky survey bright source catalogue, A\&A 349 (1999) 389

[4] R. D. Saxton et al., The first XMM-Newton slew survey catalogue: XMMSL1, A\&A 480 (2008) 611

[5] J. B. Stephen et al., Using the ROSAT catalogue to find counterparts for unidentified objects in the first Fermi/LAT catalogue, MNRAS 408 (2010) 422

[6] N. Gehrels et al., The Swift Gamma-Ray Burst mission, ApJ 611 (2004) 1005

[7] F. Massaro et al., Unveiling the nature of unidentified Gamma-Ray sources. II. Radio, infrared, and optical counterparts of the Gamma-Ray blazar candidates, ApJS 206 (2013a) 13

[8] I. Prandoni et al., The ATESP radio survey. II. The source catalogue, A\&AS 146 (2000) 41

[9] A. Schwope et al., The ROSAT Bright survey: II. Catalogue of all high-galactic latitude RASS sources with PSPC countrate CR $>0.2 s^{-1}$, AN 321 (2000) 1

[10] E. L. Wright et al., The Wide-field Infrared Survey Explorer (WISE): mission description and initial on-orbit performance, AJ 140 (2010) 1868

[11] A. Paggi et al., Optical spectroscopic observations of gamma-ray blazar candidates. I. Preliminary results, AJ 147 (2014) 112

[12] T. Murphy et al., The second epoch Molonglo Galactic Plane Survey: compact source catalogue, MNRAS 382 (2007) 382

[13] G. Tsarevsky et al., A search for very active stars in the Galaxy. First results, A\&A 438 (2005) 949

[14] N. Masetti et al., BL Lacertae identifications in a ROSAT-selected sample of Fermi unidentified objects, A\&A 559 (2013) A58

[15] E. K. Mahony et al., The RASS-6dFGS catalogue: a sample of X-ray selected AGN from the 6dF Galaxy Survey, MNRAS $\mathbf{4 0 1}$ (2010) 1151

[16] F. Massaro et al., BL Lac candidates for TeV observations, ApJS 2067 (2013b) 16

[17] T. Hassan et al., Gamma-ray active galactic nucleus type through machine-learning algorithms, MNRAS 428 (2013) 220

[18] Y. Takeuchi et al., Multiband diagnostics of unidentified 1FGL sources with Suzaku and Swift X-Ray observations, ApJS 208 (2013) 25

[19] J. B. Hutchings and L. Bianchi, A catalog of 19,100 quasi-stellar object candidates with redshift 0.5-1.5 AJ 140 (2010) 1987

[20] F. Mantovani et al., Flux density measurements of a complete sample of faint blazars, A\&A 533 (2011) A79

[21] F. E. Bauer et al., RBSC-NVSS sample. I. Radio and optical identifications of a complete sample of 1556 bright X-Ray sources, ApJS 129 (2000) 547

[22] S. Piranomonte et al., The sedentary survey of extreme high-energy peaked BL Lacs. III. Results from optical spectroscopy, $A \& A 470$ (2007) 787 